\title{
«Una lettrice formidabile»: Natalia Ginzburg e la casa editrice Einaudi
}

"Une lectrice formidable ": Natalia Ginzburg et la maison d'édition Einaudi

"An Amazing Reader": Natalia Ginzburg and the Einaudi Publishing House

\section{Laura Antonietti}

\section{OpenEdition}

\section{Journals}

Edizione digitale

URL: https://journals.openedition.org/cei/8590

DOI: $10.4000 /$ cei.8590

ISSN: 2260-779X

\section{Editore}

UGA Éditions/Université Grenoble Alpes

\section{Edizione cartacea}

ISBN: 978-2-37747-257-4

ISSN: $1770-9571$

Notizia bibliografica digitale

Laura Antonietti, ««Una lettrice formidabile»: Natalia Ginzburg e la casa editrice Einaudi», Cahiers d'études italiennes [Online], 32 | 2021, online dal 01 mars 2021, consultato il 16 septembre 2021. URL: http://journals.openedition.org/cei/8590 ; DOI: https://doi.org/10.4000/cei.8590

Questo documento è stato generato automaticamente il 16 septembre 2021. 


\title{
«Una lettrice formidabile»: Natalia Ginzburg e la casa editrice Einaudi
}

\author{
"Une lectrice formidable »: Natalia Ginzburg et la maison d'édition Einaudi \\ "An Amazing Reader": Natalia Ginzburg and the Einaudi Publishing House
}

\section{Laura Antonietti}

1 Gli stretti legami con la casa editrice Giulio Einaudi segnano in mondo costante e profondo sia il percorso creativo, in qualità di autrice di narrativa, di saggistica e di teatro ${ }^{1}$, sia l'attività editoriale di Natalia Ginzburg, che inizia nella seconda metà degli anni Trenta e si protrae in modo pressoché continuativo ${ }^{2}$ fino all'anno prima della sua morte, nel 1991. Come collaboratrice esterna, traduce il primo volume di À la recherche du temps perdu di Marcel Proust ${ }^{3}$. Nel 1944, dopo la morte per mano dei nazifascisti del marito Leone Ginzburg, tra i fondatori nel 1933 della casa editrice insieme allo stesso Giulio Einaudi ${ }^{4}$, Natalia viene assunta dapprima nella sede romana e, a partire dal 1945, in quella torinese della casa editrice e svolge, a vario titolo, nel corso degli anni, diverse mansioni: traduttrice, coordinatrice e correttrice di traduzioni; responsabile dell'editing e della correzione delle bozze, così come dei rapporti con gli autori e con $\mathrm{i}$ collaboratori esterni, lettrice di manoscritti di narrativa italiana e straniera. Ed è proprio su quest'ultimo aspetto, la lettura editoriale, che si vorrebbe focalizzare l'attenzione in questa sede.

2 Prodotto di una scrittura professionale che si caratterizza per la sua destinazione e circolazione interna, il parere di lettura rappresenta un passaggio fondamentale del percorso decisionale che porta alla pubblicazione di un'opera e quindi alla costruzione della proposta di una casa editrice, che ha proprio nel catalogo la sua più grande ricchezza e la sua eredità più significativa: non si esagera quindi definendo l'insieme dei pareri di lettura come una parte fondamentale del patrimonio storico dell'editore e lo studio di questi come passaggio essenziale per comprendere il sistema letterario ed editoriale italiano del ' 900 .

Il gruppo di collaboratori di Einaudi costituiva una folta schiera di «letterati editori» ${ }^{5}$, espressione consolidatasi negli ultimi anni per indicare un fondamentale aspetto della 
cultura letteraria italiana del Novecento: la diretta partecipazione degli hommes de lettres ai processi produttivi dell'attività editoriale. Limitandoci all'immediato dopoguerra, tra i collaboratori a vario titolo di Einaudi possiamo individuare Cesare Pavese, Elio Vittorini, Italo Calvino, Natalia Ginzburg, Franco Lucentini, Carlo Fruttero e molti altri. I pareri di lettura da loro redatti debbono essere dunque letti non come semplici documenti di lavoro, ma come esempi della più alta metaletteratura, dove alcune tra le figure chiave della storia letteraria del Novecento italiano giudicano le prove narrative di altri scrittori o aspiranti tali, mentre si confrontano, inevitabilmente, anche con la propria poetica e idea di letteratura. La scrittura editoriale viene in tal senso a rappresentare un micro-genere letterario ${ }^{6}$ specifico e complesso, che merita di essere studiato attraverso appositi strumenti, e non una semplice attività secondaria e strumentale, secondo un tradizionale pregiudizio che separa, prediligendole, l'opera letteraria e l'attività creativa dello scrittore dal suo lavoro all'interno di una casa editrice. Tale pregiudizio spiega una serie di lacune critico-bibliografiche relative a diversi personaggi chiave dell'editoria del Novecento, che possono essere definiti, secondo una fortunata espressione coniata da Gian Carlo Ferretti, come veri e propri «protagonisti nell'ombra»?

4 Natalia Ginzburg risulta essere una figura triplicemente in ombra: in primo luogo, secondo la definizione di Ferretti, in quanto costituisce uno di quei personaggi che attendono di essere studiati in modo organico e completo per il loro specifico e fondamentale lavoro nell'editoria italiana del Novecento. Emblematico in tal senso il fatto che ancora oggi non esista uno studio monografico dedicato a tale aspetto della sua attività intellettuale ${ }^{8}$, nonostante il ruolo chiave da lei rivestito per più di quarant'anni all'interno dell'Einaudi.

5 In secondo luogo, Natalia Ginzburg appare come una figura in ombra in quanto donna tout court: per tale condizione, «si pone dentro confini di marginalità, separatezza a fronte di un protagonismo tutto maschile» ${ }^{9}$ lasciando ad oggi la questione della presenza delle donne nel mondo editoriale e del contributo delle singole figure ancora da indagare e approfondire ${ }^{10}$. In terzo luogo, sintomatico anche il fatto che Ginzburg entri nel mondo dell'editoria come traduttrice, ossia con il ruolo che più tipicamente ed emblematicamente costituisce un lavoro nell'ombra e che, d'altronde, definisce in larga misura la presenza femminile (spesso non riconosciuta) ${ }^{11}$ nel mercato editoriale fra gli anni Trenta e Quaranta:

Le traduttrici attraverso il loro lavoro, che rappresenta soprattutto una fonte di guadagno, avviano un processo di affermazione, legittimazione e partecipazione dentro una casa editrice (pubblicano loro stesse opere saggistiche o altro). Contestualmente le letterate delineano le trasformazioni di un sistema culturale che progressivamente favorisce l'entrata dell'intellettualità femminile e delinea i connotati di protagoniste, «letterate editrici», che cominciano con i loro ricchi carteggi a costruire una storia che ancora deve essere raccontata ${ }^{12}$.

6 Si potrebbe infine ipotizzare un quarto motivo, da imputare al carattere timido e schivo di Ginzburg: la sua predisposizione a non apparire e a mantenere una posizione defilata (accompagnata da un costante e dichiarato senso di inadeguatezza), è testimoniata in diversi punti dell'epistolario ${ }^{13} \mathrm{e}$ nei verbali delle riunioni editoriali del mercoledì, dove raramente prende la parola.

7 A dispetto dunque delle lacune critico-bibliografiche che circondano la sua attività editoriale, Natalia Ginzburg ha assunto, nella costruzione della proposta letteraria di Einaudi, un ruolo fondamentale. Il suo peso all'interno della casa editrice aumenta nel 
corso degli anni, a partire dalla conclusione del conflitto: secondo l'organigramma della casa editrice stabilito nel 1945, Natalia Ginzburg risulta responsabile, in qualità di viceconsulente, insieme a Cesare Pavese ed Elio Vittorini, in qualità di consulenti, delle collane «Narratori contemporanei» (poi, dal 1947, «Coralli»), "Giganti», «Poeti» e «Narratori stranieri tradotti» ${ }^{14}$. È proprio sul dopoguerra che si vorrebbe focalizzare l'attenzione in questa sede, parlando di Ginzburg in qualità di lettrice editoriale di narrativa italiana, nel pieno di quegli anni che costituiscono «l'età eroica dei vecchi amici $»^{15}$ : in questo lasso di tempo la figura di Natalia, da presenza secondaria e defilata, cresce di importanza fino a diventare uno dei riferimenti principali per la narrativa Einaudi. A proposito di quel periodo, in un'intervista del 1990 di Marino Sinibaldi riportata nel volume È difficile parlare di sé, Ginzburg affermava:

[La Resistenza] aveva unito, c'era un senso di unione, di solidarietà, all'inizio. C'era un senso di profonda amicizia tra le persone, che era incominciata nel tempo della Resistenza e continuava. C'era alla casa editrice Einaudi un senso di ripresa molto forte, e molto bello; eravamo molto legati, discutevamo moltissimo, pieni di voglia di fare delle cose ${ }^{16}$.

Mentre Vittorini lavorava dalla sede milanese dell'editore, Ginzburg svolgeva la propria attività a Torino, fianco a fianco con Cesare Pavese, suo amico, maestro e grande promotore, presso Einaudi e presso diverse riviste, della sua opera come autrice ${ }^{17}$ : essi condividevano la medesima concezione del lavoro editoriale, quella che la critica pavesiana definisce 'etica del fare'. Scriveva a tal proposito Cesare Pavese a Vittorini il 5 ottobre 1948: «rivedo manoscritti, correggo bozze: io insomma faccio i libri» ${ }^{18}$, gettando luce sull'attento e scrupoloso lavoro redazionale che tanto Pavese quanto Natalia avevano appreso ed ereditato da Leone Ginzburg.

La consonanza di idee e di gusto letterario presente tra i due, testimoniata da diversi pareri di lettura in cui mostrano un sostanziale accordo, porta a una sorta di abdicazione da parte di Cesare Pavese in favore di Natalia Ginzburg per quel che riguarda la narrativa contemporanea: nel corso del 1948 Pavese smette infatti sostanzialmente di occuparsene per dedicarsi quasi esclusivamente alla "Collana viola», la «Collezione di studi religiosi, etnologici e psicologici». Questo passaggio di testimone è documentato in diversi punti dell'epistolario pavesiano. A titolo di esempio, possiamo citare la lettera a Carlo Muscetta, altro collaboratore di Einaudi, del 26 agosto 1948:

Ti rimando Vento caldo di Moretti col giudizio di Natalia. [...] Lo sottoscrivo e penso di non farlo. Bada che io non ho letto il libro, perché sono stufo di «balle» e trovo molto più spiritose quelle della mia «etnologica». D'or innanzi sei avvertito: della narrativa si occupa soltanto Natalia ${ }^{19}$.

È possibile poi fare riferimento alla già citata lettera a Vittorini del 5 ottobre 1948, dove Pavese afferma che «l'unica collezione che ancora m'interessa è quella etnologica, e sistematicamente rispondo ai postulanti narratori e poeti che quanto decide Natalia e, se del caso, Vittorini, per me sta bene» ${ }^{20}$. E ancora in una lettera del 17 febbraio 1949 all'agente americano Greenburger, Pavese indica Natalia Ginzburg come responsabile della narrativa italiana e francese, mentre definisce se stesso responsabile della sezione etnologica e antropologica ${ }^{21}$ : questo costituisce la «sanzione del ruolo sempre più significativo assunto da Natalia Ginzburg nella casa editrice» ${ }^{22}$.

Il distacco di Pavese dalla narrativa corrisponde quindi all'assurgere di Natalia Ginzburg a responsabile principale in tale ambito: questo cambiamento viene sancito dal nuovo organigramma della casa editrice approvato nel $1949^{23}$, in cui Natalia Ginzburg risulta responsabile (insieme a Bruno Fonzi, entrato in casa editrice nel 1948) 
dei «Coralli», dei «Supercoralli» e dei «Narratori stranieri tradotti». È importante sottolineare anche come, nello stesso periodo, emerga un'altra figura di massima importanza per la costruzione del catalogo einaudiano (in particolare per la narrativa): Italo Calvino, che entra a far parte dell'organico nel 1950, dopo un periodo di collaborazione saltuario. Egli diventerà, nel corso degli anni, uno dei principali interlocutori di Ginzburg, sulle opere altrui e della stessa Ginzburg.

Allo scopo di entrare nel vivo del lavoro di lettura editoriale, si prendono in questa sede in esame alcune carte d'archivio, che rappresentano un campione limitato ma al tempo stesso emblematico: Ginzburg appare come una lettrice assidua e instancabile, attenta e scrupolosa, sincera e imparziale, diretta se non a tratti dura ma, al contempo, capace di consigliare i giovani autori che si rivolgevano all'editore o di prendersi a cuore la pubblicazione delle opere che l'avevano colpita. Si possono ritrovare le qualità appena descritte nelle parole dei collaboratori einaudiani: Cesare Pavese scriveva a Silvio Micheli, in una lettera del 29 dicembre 1945 di tenere conto del giudizio di Ginzburg, "perché è una donna che si entusiasma difficilmente»" ${ }^{24}$ ancora a Carlo Musso, l'8 gennaio 1947 «è una donna incapace di mentire in fatto di gusto» ${ }^{25}$. Giulio Einaudi, nel già citato Ė difficile parlare di sé, sottolinea la schiettezza e l'onestà intellettuale della Ginzburg, definendola «perno del lavoro editoriale negli anni dopo la Liberazione» e «coscienza critica della casa editrice»: «se c'è qualcosa che non gli piace lo dice subito, non è come quelli che fan finta di niente. Lei lo dice, lo sbraita, lo urla, lo dice alla televisione se può, alla radio» ${ }^{26}$. Ed è di nuovo l'editore che descrive Ginzburg, in Colloquio con Giulio Einaudi, come «una lettrice formidabile. Dà consigli ai giovani scrittori, ne sposa i libri, li propone con insistenza» ${ }^{27}$.

Esemplare risulta in tal senso il caso di L'Agnese va a morire, romanzo partigiano autobiografico di impianto neorealista scritto da Renata Viganò, pubblicato da Einaudi nel 1949 e vincitore, nel medesimo anno, del Premio Viareggio. Scrive Ginzburg all'autrice, in una lettera del 27 ottobre 1948 che fa emergere, tra l'altro, il suo modo di lavorare trasparente e onesto, non condizionato nella lettura né da presentazioni né da raccomandazioni esterne:

È molto bello. Il dattiloscritto era sul mio tavolo da un pezzo, senza nessuna lettera accompagnatoria: io avevo un mucchio di manoscritti, ed ho pescato il suo a caso nel mucchio. Un bel romanzo. Tra i migliori romanzi partigiani che ho letto ${ }^{28}$.

Lo stesso entusiasmo emerge con convinzione anche nel parere di lettura, non datato, di Ginzburg:

Un bellissimo romanzo partigiano. Magnifico stile, misurato, sobrio, magnifici effetti di paesaggio. Tra i migliori libri sulla resistenza che si possono leggere. È la storia di una staffetta partigiana, una contadina. [...] La resistenza è vista proprio con gli occhi dei contadini. Da farsi, da farsi, da farsi ${ }^{29}$.

Da quanto risulta dalle carte d'archivio, questo è uno dei pochi casi in cui un redattore einaudiano riesce ad imporre all'editore la pubblicazione di un'opera senza che vengano effettuate altre letture editoriali (normalmente tre): il libro uscì infatti nel giro di pochi mesi. L'entusiasmo di Ginzburg può essere ricondotto a diversi fattori, molti dei quali è possibile mettere in relazione con la sua opera di scrittrice, percorrendo così una delle strade più interessanti che gli studi sui letterati editori offrono: analizzare il legame tra la loro attività creativa e quella editoriale. L'anno precedente, nel 1947, Ginzburg pubblicava il romanzo breve È stato così, definito dalla critica coeva il frutto del suo periodo neorealista ${ }^{30}$. Nella già citata intervista contenuta 
in È difficile parlare di sé, alla domanda sulla poetica e lo stile neorealisti (proprio in relazione a È stato cosi) Ginzburg rispondeva:

Mah, allora mi attirava; mi sembrava che volevo il neorealismo. Insomma, volevo il neorealismo. Insomma, volevo sfuggire a quella che era stata la letteratura negli anni del fascismo, cioè lontana, distante [...] lontana dai fatti della vita [...] il neorealismo mi sembrava che fosse l'avvicinarsi alla vita, il penetrare nella vita, nella realtà ${ }^{31}$.

Ginzburg, nel suo parere di lettura su L'Agnese va a morire, non faceva riferimento diretto al neorealismo in quanto, in generale, risulta scarsamente interessata a collocare e quindi giudicare un'opera in un genere o in una corrente letteraria, ma pare evidente che il romanzo rispondesse a quello che la stessa Ginzburg ammetteva essere stato, in quel periodo, il suo gusto letterario come scrittrice e, in seconda battuta, come editrice.

17 Vale la pena sottolineare come tuttavia, successivamente, il neorealismo (la sua poetica e le sue implicazioni politiche), sia stato oggetto di un processo di rivalutazione e di rimessa in discussione da parte di Natalia Ginzburg. Nelle pagine di Lessico famigliare, la principale critica che muoveva al neorealismo era quella di aver confuso la poesia, intesa nel senso ampio di letteratura, e la politica:

Era, il dopoguerra, un tempo in cui tutti pensavano d'essere dei poeti, e tutti pensavano d'essere dei politici; tutti s'immaginavano che si potesse e si dovesse anzi far poesia di tutto, dopo tanti anni in cui era sembrato che il mondo fosse ammutolito e pietrificato e la realtà era stata guardata come di là da un vetro, in una vitrea, cristallina e muta immobilità. Romanzieri e poeti avevano, negli anni del fascismo, digiunato, non essendovi intorno molte parole che fosse consentito usare; e i pochi che ancora avevano usato parole le avevano scelte con ogni cura nel magro patrimonio di briciole che ancora restava. [...] Ora c'erano di nuovo molte parole in circolazione, e la realtà di nuovo appariva a portata di mano; perciò quegli antichi digiunatori si diedero a vendemmiarvi con delizia. E la vendemmia fu generale, perché tutti ebbero l'idea di prendervi parte; e si determinò una confusione di linguaggio fra poesia e politica, le quali erano apparse mescolate insieme. [...] C'erano allora due modi di scrivere, e uno era una semplice enumerazione di fatti, sulle tracce d'una realtà grigia, piovosa, avara, nello schermo d'un paesaggio disadorno e mortificato; l'altro era un mescolarsi ai fatti con violenza e delirio di lagrime, di sospiri convulsi, di singhiozzi. [...] Ne conseguì un disgusto di poesia $\mathrm{e}$ parole, così forte che incluse anche la vera poesia e le vere parole, per cui alla fine ognuno tacque, impietrito di noia e di nausea. Era necessario tornare a scegliere le parole, a scrutarle per sentire se erano false o vere, se avevano o no vere radici in noi, o se avevano soltanto le effimere radici della comune illusione ${ }^{32}$.

18 Ed è ancor all'interno di una delle interviste contenute in È difficile parlare di sé che Ginzburg traccia l'allontanamento del suo percorso di scrittrice dal neorealismo e da una concezione impegnata della letteratura:

Le parole «impegno» e «disimpegno» c'erano continuamente, circolavano continuamente nell'immediato dopoguerra. Io a un certo momento ho pensato che volevo il disimpegno... non che lo volevo, ma che era assolutamente necessario, indispensabile per un romanziere. Che un romanziere non doveva porsi il dovere di cercare di portare dei miglioramenti alla società, ma invece semplicemente scrivere meglio possibile i suoi romanzi. E di questo sono sicura ${ }^{33}$.

Per tornare dunque al caso dell'Agnese va a morire, risulta evidente (nonostante a posteriori, rileggendo e reinterpretando il proprio percorso letterario e intellettuale, essa stessa proclami una volontà di disimpegno) lo sforzo di Ginzburg, come autrice e come editrice, di partecipare al processo collettivo di rielaborazione della guerra e della 
Resistenza. È importante infatti ricordare che nel 1952, viene pubblicato Tutti i nostri ieri, il romanzo di Ginzburg in cui la Resistenza è più presente: la memoria nella chiave della memoria storica e individuale della Resistenza, della maturazione politica di una generazione, diventa, a partire proprio da questo romanzo, un elemento sempre più presente nella narrativa dell'autrice ${ }^{34}$.

Se è poi vero che anche da un punto di vista stilistico è possibile notare una certa consonanza tra Ginzburg e Viganò (uno stile «misurato, sobrio»: scarno, asciutto, semplice, concentrato), Ginzburg si mostra tuttavia capace di andare al di là del proprio gusto e delle proprie idiosincrasie nel giudizio sulla pubblicabilità di un'opera. È il caso, ad esempio, di Renata della vita di David Invrea. Scriveva Ginzburg il 26 gennaio 1950 nel relativo parere di lettura:

Romanzo a me profondamente antipatico ma pieno di intelligenza, notevole storia di una prostituta, personaggio di prostituta nuovo; in coscienza devo dire che questo romanzo merita di essere stampato. Ha un notevole interesse di lettura. È odioso, comunque, perché è scritto in uno stile sofisticato e fuligginoso, e perché i personaggi sono detestabili, ma veri; una Torino di ladri e di prostitute molto vera e nuova. Il romanzo presentava questi caratteri già nella prima stesura, ma in forma caotica e stravolta. Ora l'ha liberato di tutti gli orrori e gli errori che c'era nella prima stesura e in coscienza direi di sì benché a malincuore ${ }^{35}$.

Il romanzo non verrà poi pubblicato, a causa del no di Vittorini e del sì, ma con riserva, di Pavese e della stessa Ginzburg. In altri casi, lo stile troppo «almanaccato», «sofisticato e fuligginoso» di Invrea, come per il romanzo Giordano e la paura, sarà giudicato da Ginzburg un ostacolo alla pubblicabilità e alla leggibilità stessa dell'opera. Scrive Ginzburg nel parere di lettura non datato (ma probabilmente risalente ai primi mesi del 1947):

È un bel romanzo. Lo frega la religione. Tutte le volte che viene fuori la monaca è una scocciatura. C'è poi un netto stacco fra il personaggio principale e un altro personaggio di scorcio che analizza e molte volte interviene con toni troppo almanaccati, e disturba.

Sono d'accordo sulla trovata stilistica del linguaggio dialettale e analitico, ma mi pare che il tono analitico doveva essere rozzo, e non almanaccato. È molto bello quando la moglie lo va a trovare in prigione.

Il personaggio principale, tutto sommato, mi pare valido. Bella la fine. Siccome c'è qualcosa che decisamente non quadra, direi di non farlo. [...] credo che quel qualche cosa che a noi non va, lo renda ostico alla lettura di un pubblico di non-scrittori, cioè di persone meno disposte ad accogliere quanto di bello c'è in questo aspro terreno ${ }^{36}$.

Pur riconoscendo il valore dell'opera al di là del proprio gusto personale, sono quindi le ragioni editoriali a prevalere. E sono sempre ragioni editoriali che spesso motivano allo stesso tempo il rifiuto, ma anche l'invito a seguire un determinato autore considerato talentuoso ma non ancora maturo poeticamente, nella speranza che possa in un secondo momento dare risultati migliori. È il caso di Vento caldo di Ugo Moretti. Il parere di lettura di Ginzburg viene riportato da Pavese nella già citata lettera a Carlo Muscetta del 26 agosto 1948:

È la storia di uno che fa un po' tutti i mestieri, poi va in guerra e torna a Roma a rifare tutti i mestieri. C'è un bell'ambientino di pittori e straccioni e pederasti, che è la cosa più felice del libro. Il libro ha dei difetti grossi come case; è scritto da uno molto in gamba, è pieno di trovate geniali ma è sovrabbondante, smodato, poi la storia d'amore che dovrebbe essere il centro del libro non sa di niente assolutamente. E poi non c'è nessuna ragione seria perché il libro cominci a un dato punto e finisca a un dato punto; per non parlare della conclusione. [...] Secondo me 
non è da fare; si capisce che l'autore non bisogna perderlo di vista perché domani può darsi che scriva un bel libro ${ }^{37}$. suoi giudizi entra nel merito dei testi a diversi livelli: la struttura e il ritmo narrativo, il sistema dei personaggi e la loro delineazione, l'ambiente fisico e sociale, la lingua e lo stile. Ginzburg mantiene lo stesso atteggiamento analitico nella corrispondenza con gli autori, con cui instaura sovente un vero e proprio dialogo, muovendo loro critiche precise e dando loro consigli. È il caso di Sergio Antonielli con Il campo 29, che viene qui portato ad esempio perché da un lato mostra la natura dialogica e collegiale del processo decisionale einaudiano, dall'altro il carattere costruttivo e maieutico dell'atteggiamento di Ginzburg. In un documento non datato (ma risalente alla prima metà del 1948) viene riferito il parere di lettura di Ginzburg, insieme a quello, in questa sede non riportato, di Pavese:

Un libro notevole. La storia di un campo di prigionieri in India: molto vivo il paesaggio; un senso preciso e fisico della vita di prigionia. Stilisticamente felice. Nessuna, o quasi, valutazione politica: il che secondo me è un difetto, perché $\mathrm{i}$ personaggi ne risultano a una dimensione sola. Lento e non divertente come lettura, ma certo notevole. Chiediamo il parere di Vittorini ${ }^{38}$.

Non c'è traccia del parere di Vittorini, ma doveva essere fortemente indeciso se Ginzburg, il 7 luglio, gli scrive:

Abbiamo deciso di non fare Il Campo 29 di Antonielli perché non c'era entusiasmo da nessuna parte. Tutti l'abbiamo trovato buono, ma noioso, e sarebbe stato di difficile vendita. Siccome anche tu eri fra il sì e il no, abbiamo stabilito di no ${ }^{39}$.

Il giorno seguente Ginzburg scrive all'autore:

Siamo lieti di avere avuto occasione di leggere il Suo manoscritto Il Campo 29, che ci è sembrato non solo un documento notevole di vita vissuta, ma anche un'opera pregevole dal punto di vista letterario. Tuttavia, considerandolo sotto l'aspetto editoriale, abbiamo gravi dubbi sulle sue possibilità di successo. L'eccessiva lentezza nel raccontare, la scarsa drammaticità, ne rendono poco agevole la lettura; e noi abbiamo l'impressione che un movimento più rapido, una narrazione più succinta, avrebbe senz'altro giovato, anche poeticamente, al suo lavoro. Inoltre mentre il paesaggio e il senso della vita di prigionia sono assai vivi ed efficaci, meno vivi e più convenzionali, meno approfonditi, ci sono sembrati i personaggi.

Le restituiamo il manoscritto [...]. Riteniamo ch'ella potrebbe forse rivolgersi con fortuna alla Casa Editrice De Silva [...] che ha una collana di documenti di attualità nella quale ci sembra che il Suo libro potrebbe rientrare felicemente ${ }^{40}$.

Pur nell'apprezzamento dell'opera, il libro viene dunque rifiutato per un insieme di ragioni editoriali (come nel caso di Invrea, si tratta di preoccupazioni per la leggibilità e quindi la vendibilità del testo) e poetiche (la scarsa drammaticità e la lentezza del racconto). La casa editrice De Silva aveva pubblicato l'anno precedente Se questo è un uomo di Primo Levi: indirizzare l'autore verso un editore ritenuto più adatto pare, soprattutto di fronte al rifiuto, un ulteriore sforzo maieutico, come già evidenziato da Giulia Iannuzzi ${ }^{41}$.

La preoccupazione per la perdita in termini di evidenza narrativa e di ritmo nel racconto, a vantaggio dell'aspetto documentario (è possibile vedere come Ginzburg parli di «documento» a più riprese in riferimento al Campo 29) o analitico, emerge nella lettura di un altro romanzo del medesimo autore, La dinastia, inviato in lettura a Einaudi nell'autunno del 1950 (e uscito poi presso Rizzoli nel 1952). Scrive Ginzburg in una lettera all'autore il 28 novembre 1950: 
Ho letto il Suo romanzo La dinastia e lo passo agli altri consulenti. Ma Le accenno intanto quello che ne penso io. Ricordo bene Il campo 29, e ne ricordo le serie qualità narrative, che ho ritrovato del resto in questo Suo secondo lavoro. Credo che lei possa arrivare a fare qualcosa di bello. Però mi sembra che, nella Dinastia, ci sia un difetto sostanziale: mi sembra che Lei descriva invece di inventare, e che i personaggi risultino morti e incoerenti, proprio perché vittime d'un'analisi troppo scavata e minuziosa. I pochi dialoghi suonano falsi e forzati; e l'azione, troppo diffusamente giustificata, appare spesso ingiustificabile. Il romanzo è pieno d'intelligenza; è carico di possibilità, non realizzate e spese male. A me è sembrato molto interessante e benché lo ritenga poeticamente non realizzato lo passo in lettura ai miei colleghi di lavoro ${ }^{42}$.

L'azione narrativa deve essere evidente in se stessa, senza che l'autore debba intervenire per giustificarla o analizzarla, pena l'artificiosità dei dialoghi o l'innaturalezza dei personaggi. È possibile fare riferimento, come ulteriore esempio, al parere su Icaro e Petronio di Elio Bartolini (Mondadori, 1950), contenuto in una lettera del 25 marzo 1949 a Vittorini:

Ho letto Icaro e Petronio e questa volta non sono d'accordo con te e neppure Fonzi: trovo che si legge con grande fatica, io sono arrivata a stento alla fine: mi pare che continuamente ragioni invece di raccontare, e ragioni a freddo in un modo indigesto e pesante. Fonzi pensa così anche lui. [...]. Mi pare che non sarebbe un bel corallo, non si vede niente, io non vedo niente: vedo uno che va e viene col camion, ma non mi basta. Comunque, sarà Giulio a decidere: magari si potrebbe darlo a un altro lettore ${ }^{43}$.

Appare dunque chiaro come, per Ginzburg, la realizzazione poetica di un'opera passi inevitabilmente attraverso la piena realizzazione della dimensione diegetica. Pienezza che, nel caso di Antonielli, verrà raggiunta, secondo la redazione Einaudi, con La tigre viziosa, pubblicata nei «Gettoni» di Elio Vittorini, nel 1954.

Si porta in questa sede un ultimo caso emblematico, in quanto in esso emergono gran parte delle caratteristiche della lettrice Natalia Ginzburg che abbiamo visto delinearsi nei documenti finora proposti: la sua franchezza e schiettezza, l'attitudine maieutica e il sincero interessamento per gli autori (anche nella prospettiva di un loro futuro editoriale presso Einaudi), la volontà di andare a fondo nelle questioni letterarie $\mathrm{e}$, infine, i legami con la sua poetica come autrice. Nel parere di lettura non datato su La morte non si è seduta sulle foglie secche di Rosita Fusé, Ginzburg scrive:

Questo manoscritto della Fusé è notevole: ma non ha il corpo di un romanzo, è un'esile novella diffusa lungo 150 pagine. Ci sono delle cose molto belle e delle pagine scolorite e convenzionali; non ci sono goffaggini o stranezze o sbavature liricheggianti, ma piuttosto il difetto della Fusé è quello di tenersi troppo legata a schemi convenzionali e letterari. Il difetto principale sta nei dialoghi: questi straccioni delle baracche si dicono: «non so ciò che posso fare per lei» e «fra voi donne vi potrete sempre intendere». Non so come dovrebbero parlare, ma non così. Ma ha molta misura e sa scrivere. Sono sicura che è una scrittrice intelligente e forse importante e da tenersi d'occhio. Ma non le pubblicherei questo, mi sembra che possa fare di meglio. È la storia di una ragazza delle baracche che fa un figlio, e tutte le donne la odiano, poi il figlio muore e allora la soccorrono. I personaggi sono tutti senza faccia, meno una vecchia, ma ci sono scene d'amore molto belle, di grande misura. Sono sicura che è una persona che bisogna incoraggiare a scrivere ${ }^{44}$.

31 Nella lettera all'autrice, risalente al 5 ottobre 1948, Natalia entra ancora nel merito del testo e rivolge alla Fusé diversi consigli e critiche:

Cara signorina,

ho sentito parlare molto di Lei e quindi ho l'impressione di conoscerla e le scrivo 
con assoluta franchezza. Sono convinta che lei ha delle possibilità narrative e molto serie [...] sul manoscritto ho delle riserve che adesso cercherò di spiegare. La riserva principale è questa: ci sono delle persone - lei e anch'io - che non sanno raccontare bene se non quello che conoscono a fondo: non sanno inventare se non quanto è molto a portata di mano; altri scrittori riescono a inventare sul niente, ma è un altro modo di scrivere. Dei tipi come me o come lei non possono concedersi che degli angoli visuali molto piccoli: non importa: soltanto bisogna saperlo e lavorare solo in questo senso. Le sue baracche non hanno nessuna evidenza poetica: sono pallide baracche, uno sfondo nebbioso con vaghe intenzioni sociali e realistiche [...] ma non hanno una vera vitalità. Quello che è vitale nel suo romanzo è la storia intima di alcuni personaggi e di alcuni rapporti [...] Un'altra riserva si riferisce all'estensione: lei avrebbe potuto dire tutto in 14 pagine invece ce ne sono 150 . Questo proprio perché l'impalcatura da romanzo che lei gli ha dato è molto dubbia $\mathrm{e}$ i suoi valori sono tutti estranei a questa impalcatura. Spero che abbia capito quello che ho voluto dire: che lei certo ci darà un romanzo migliore; se non ne fossi certa, non avrei scritto così a lungo e così schiettamente. Non deve dunque scoraggiarsi $\mathrm{e}$ neppure ritenere che sia un'esperienza andata a vuoto: uno prova e riprova finché scopre il suo vero mondo narrativo. Se mi permette di darle un consiglio, io le direi di inventare il meno possibile, di tenersi il più vicino possibile alla realtà della sua stessa vita ${ }^{45}$. abbiamo visto sinora adottare a Ginzburg per accettare o rifiutare un romanzo: i giudizi sulla misura del racconto (che avrebbe beneficiato di una «narrazione più succinta» nel caso del Campo '29, «sovrabbondante, smodato» nel giudizio su Vento caldo) e sul suo ritmo (si veda la «lentezza del raccontare» rilevata nel Campo '29), l'attenzione per la dimensione diegetica e la piena realizzazione poetica (si ricorda la «scarsa drammaticità» e l'«azione spesso ingiustificabile» dei romanzi di Antonielli o «la storia d'amore che dovrebbe essere il centro del libro» e che invece «non sa di niente assolutamente» e l'assenza di una «ragione seria perché il libro cominci a un dato punto e finisca a un dato punto del romanzo» nel caso di Vento caldo o, ancora, l'impressione che Bartolini in Icaro e Petronio «ragioni invece di raccontare, e ragioni a freddo in un modo indigesto e pesante»), l'importanza assegnata ai personaggi e, in particolar modo, al loro modo di esprimersi e di dialogare, frutto talvolta di un eccesso di analisi («il tono analitico doveva essere rozzo, e non almanaccato» scrive a proposito di Giordano e la paura; i personaggi risultano «morti e incoerenti, proprio perché vittime d'un analisi troppo scavata e minuziosa. I pochi dialoghi suonano falsi e forzati» nota a proposito de La dinastia). Emerge qui, tuttavia, un altro elemento, fondamentale nella prospettiva dell'analisi delle relazioni tra scelte editoriali e poetica individuale: la necessità di raccontare solo e soltanto ciò che si conosce bene, aderendo il più possibile alla realtà della propria vita. Se è la Ginzburg editrice che scrive, è la Ginzburg scrittrice che si affaccia tra le righe, in una sorta di slancio empatico e d'immedesimazione. Quasi con le stesse parole descrive infatti la propria scrittura nella già citata intervista condotta da Marino Sinibaldi:

Io voglio il distacco: però non riesco a scrivere se non in prima persona. [...] Se non dicendo «io»: o un io inventato o un io reale, mio (cioè io stessa), ma sempre guardando le cose da un angolo solo, guardando il mondo da un angolo solo. Non mi è mai riuscito di salire sulle montagne e vedere tutto dall'alto, non mi è mai riuscito ${ }^{46}$.

Ed è ancora la stessa Ginzburg che nel saggio Il mio mestiere, parlando di sé e della sua scrittura, afferma: 
C'è il pericolo di truffare con parole che non esistono davvero in noi, che abbiamo pescato su a caso fuori di noi e che rimettiamo insieme con destrezza perché siamo diventati piuttosto furbi. C'è il pericolo di fare i furbi e truffare. È un mestiere è abbastanza difficile, lo vedete, ma il più bello che ci sia al mondo ${ }^{47}$.

Nella Nota dell'autrice ai Cinque romanzi brevi (Einaudi, 1964), un importante documento di poetica, Natalia Ginzburg, all'indomani del successo di Lessico famigliare, riflette sul proprio processo creativo e traccia il suo percorso autoriale-letterario, figurandolo come un itinerario verso la "pura, nuda, scoperta e dichiarata memoria» ${ }^{48}$ : è dunque nella piena accettazione e nell'appropriazione letteraria del proprio «angolo così ristretto», nella consapevolezza di poter «raccontare soltanto quello che si conosce, quello che si conosce dal di dentro» allontanandosi così il più possibile dal mondo dell'invenzione, nell'approdo all'«io» e nel superamento dell'«orrore dell'autobiografia $»^{49}$, in definitiva, nella narrativa di pura memoria, che l'autrice dichiara di trovare la propria felicità creativa:

Così arrivai alla pura memoria: vi arrivai a passi di lupo, prendendo vie traverse, dicendomi che le fonti della memoria erano quelle a cui non dovevo mai bere, l'unico luogo al mondo in cui dovevo rifiutarmi di andare. E non so se scriverò ancora altri libri: ma so che se scrivessi ancora dovrei ritrovarmi in quello stato di assoluta e pura libertà ${ }^{50}$.

In queste riflessioni, in cui emerge una sostanziale consonanza di idee tra l'autrice e l'editrice, Natalia Ginzburg traccia il suo percorso verso una nuova forma di realismo, che definiamo in questa sede realismo autobiografico, dove l'«io», la memoria e l'adesione alla realtà della propria vita acquisiscono progressivamente spazio e dignità letteraria.

Da queste pagine emerge una figura intellettuale complessa ed eccezionale nella sua capacità di lettura e nel suo acume critico. Lettura editoriale e scrittura creativa sono in continuo rapporto dialettico, intrecciandosi e intersecandosi continuamente, senza tuttavia mai confondersi: grazie infatti a un rigore straordinario, Ginzburg li mantiene distinti, lasciando allo stesso tempo che essi si nutrano e si arricchiscano vicendevolmente. Per questa ragione appare fondamentale analizzare in che modo la poetica di Ginzburg autrice influenzi la sua attività di lettura; viceversa, uno studio dell'attività editoriale risulta essenziale per una piena comprensione della sua opera di scrittrice.

\section{NOTE}

1. Tutte le opere di Ginzburg furono pubblicate con Einaudi, ad eccezione di Paese di mare e altre commedie (Garzanti, 1971), Caro Michele (Mondadori, 1973) e Vita immaginaria (Mondadori, 1974). 2. In un primo tempo collaboratrice interna, il 31 dicembre 1955 Ginzburg firma un contratto di consulenza esterna con la casa editrice. La collaborazione continua nel periodo in cui l'autrice segue il secondo marito Gabriele Baldini a Londra (1959-1961); negli anni '60 i rapporti di consulenza si diradano per poi ricominciare a pieno titolo nella seconda metà degli anni '70. Per una rapida ricostruzione cronologica del lavoro editoriale di Natalia Ginzburg, si vedano 
G. Iannuzzi, Natalia Ginzburg. Una linea editorial-creativa, in G. C. Ferretti (a cura di), Protagonisti nell'ombra. Milano, Unicopli, 2012, pp. 115-133 e N. Saita, Natalia Ginzburg la fedeltà di una vita con "passo da soldato», in R. Cicala e V. La Mendola (a cura di), Libri e scrittori di via Biancamano, Casi editoriali in 75 anni di Einaudi, Milano, EDUCatt, 2009, pp. 95-124.

3. La traduzione di Du côté de chez Swann uscì presso Einaudi nel 1946, con il titolo La strada di Swann.

4. Sulla figura intellettuale di Leone e sul suo ruolo nella fondazione della casa editrice, si veda almeno A. D'Orsi, L'intellettuale antifascista: ritratto di Leone Ginzburg, Vicenza, Pozza, 2019; N. Tranfaglia (a cura di), L'itinerario culturale di Leone Ginzburg, Torino, Bollati Boringhieri, 1996; L. Ginzburg, Lettere dal confino. 1940-1943, a cura di L. Mangoni, Torino, Einaudi, 2004; L. Ginzburg, Scritti, a cura di D. Zucaro, Torino, Einaudi 2000. Si vedano egualmente le parti dedicate a tale soggetto negli studi sulla casa editrice Einaudi, in particolar modo in L. Mangoni, Pensare i libri. La casa editrice Einaudi dagli anni Trenta agli anni Sessanta, Torino, Bollati Boringhieri, 1999 e G. Turi, Casa Einaudi: libri uomini idee oltre il fascismo, Bologna, Il Mulino, 1990.

5. Espressione utilizzata per la prima volta in A. Cadioli, Letterati editori, Milano, Il Saggiatore, 1995 (ed. successive, ampliate, del 2003 e 2017).

6. M. Bricchi, La scheda di lettura come micro-genere letterario, in S. Arduini e I. Carmignani (a cura

di) Giornate della traduzione letteraria. Nuovi contributi, Roma, Iacobelli, 2010, pp. 54-66.

7. G. C. Ferretti, Protagonisti nell'ombra, Milano, Unicopli, 2012.

8. Oltre ai riferimenti nei saggi e nelle monografie riguardanti l'opera di Natalia Ginzburg scrittrice e negli apparati delle singole opere, l'attività editoriale di Ginzburg è specificatamente trattata, in aggiunta ai già citati saggi di Iannuzzi e Saita, in G. Cannì, Natalia Ginzburg alla casa editrice Einaudi. «Una redattrice pigra e incompetente?», in P. Gabrielli (a cura di), Vivere da protagoniste. Donne tra politica, cultura e controllo sociale, Roma, Carocci, 2001, pp. 159-190. Particolarmente utili alla ricostruzione dell'attività editoriale di Ginzburg sono le testimonianze contenute in N. Ginzburg, È difficile parlare di sé. Conversazione a più voci condotta da Marino Sinibaldi, a cura di C. Garboli e L. Ginzburg, Torino, Einaudi, 1999. Diversi cenni interessanti e ricchi di rimandi a materiali inediti, per quanto spesso mancanti di riferimenti bibliografici e archivistici, si trovano nella biografia scritta da S. Petrignani, La Corsara. Ritratto di Natalia Ginzburg, Vicenza, Neri Pozza, 2018. Utili anche i riferimenti contenuti nei saggi del numero monografico di «Autografo», dedicato a Natalia Ginzburg, a cura di Maria Antonietta Grignani e Domenico Scarpa (Novara, Interlinea, 2017), in particolar modo i materiali inediti pubblicati nella sezione Breviario di uno scrittore. Scritti, lettere e pareri editoriali (1944-1966), a cura di Scarpa (pp. 167-198). È possibile trovare inoltre Natalia Ginzburg menzionata nelle storie dell'editoria, nei volumi sulla storia dell'Einaudi (in particolare nei già citati studi di Mangoni e Turi) o ancora nei volumi dedicati ai colleghi einaudiani la cui attività è stata sinora maggiormente studiata (si vedano gli studi di Ferretti e Cadioli dedicati a Pavese, Calvino, Vittorini). Si trovano poi cenni sparsi al suo lavoro in Einaudi all'interno di profili biografici, libri-intervista e libri di memorie di Giulio Einaudi e altri collaboratori einaudiani (G. Einaudi, Frammenti di memoria, Milano, Rizzoli, 1988; Id., Tutti i nostri mercoledi. Interviste di Paolo Di Stefano, Bellinzona, 2001; S. Cesari, Colloquio con Giulio Einaudi, Roma / Napoli, Theoria, 1991; E. Ferrero, I migliori anni della nostra vita, Milano, Feltrinelli, 2016). Per mezzo di una trattazione approfondita dell'attività editoriale di Ginzburg, si otterrebbe un contributo eccezionale anche allo studio della sua opera di scrittrice, rileggendo la sua opera alla luce del suo lavoro editoriale, testimoniato dall'abbondanza di documenti inediti o comunque mai studiati organicamente conservati presso l'archivio storico della casa editrice (d'ora in poi $\mathrm{AE}$ ), secondo una modalità critica adottata da vari studi negli ultimi decenni, primi fra tutti quelli di Cadioli e Ferretti su altre personalità dell'editoria italiana (si veda, a titolo di esempio, il già citato A. Cadioli, Letterati editori e G. C. Ferretti, L'editore Vittorini, Torino, Einaudi, 1992; Id., Poeta e di poeti funzionario. Il lavoro editoriale di Vittorio Sereni, Milano, Il Saggiatore 1999 o ancora Id., L'editore Cesare Pavese, Torino, Einaudi, 2017). In aggiunta a quanto appena scritto, una trattazione 
critica complessiva della collaborazione editoriale di Ginzburg risulterebbe un fondamentale contributo agli studi di storia dell'editoria e in particolare su casa Einaudi e dunque su un luogo strategico per la comprensione del sistema letterario italiano nel dopoguerra.

9. L. Di Nicola, «Dalla parte dell'ombra. Donne e editoria», in Nuovi annali della Scuola speciale per archivisti e bibliotecari, anno XXVI, 2012, p. 157.

10. Un lavoro pionieristico in tal senso è stato portato avanti con la pubblicazione di Donne in editoria / Women in publishing, a cura di Roberta Cesana, «Bibliologia», voll. IX (2014) e X (2015).

11. «La presenza femminile nel mercato editoriale fra gli anni Trenta e Quaranta si definisce per lo più attraverso il lavoro di traduzione. [...] Si tratta di nomi di traduttrici che definiscono i connotati di un'intellettualità femminile attiva che comincia a configurarsi come un vero e proprio investimento culturale per le case editrici. Gli stessi letterati, Vittorini, Gadda, Montale, scelgono la traduzione come il canale per "entrare", per sostenersi economicamente, ma portano avanti tale lavoro avvalendosi delle competenze e della professionalità di donne, "traduttrici segrete" del cui lavoro essi si appropriano» (L. Di Nicola, Dalla parte dell'ombra. Donne e editoria, cit., p. 162). Si veda l'emblematico caso di Lucia Rodocanachi, intellettuale colta e poliglotta, che per anni tradusse testi per Vittorini, Gadda e Montale. Chiamata a ragione da Montale «la négresse inconnue», portò avanti per anni un lavoro non firmato (o meglio, firmato dai letterati che lo commissionavano) e ufficialmente non riconosciuto. Per ulteriori approfondimenti, si veda E. Vittorini, Si diverte tanto a tradurre? Lettere a Lucia Rodocanachi, 1933-1943, a cura di A. C. Cavallari ed E. Esposito, Milano, Archinto, 2016; F. Cotorbia (a cura di), Lucia Rodocanachi: le carte, la vita, Firenze, Società Editrice Fiorentina, 2006; G. Marcenaro, Una amica di Montale. Vita di Lucia Rodocanachi, Milano, Camunia, 1991. Al di là del caso di Lucia Rodocanachi e di quello di Natalia Ginzburg, per citare solo alcuni degli esempi più noti di figure femminili che iniziano la loro attività editoriale come traduttrici, è possibile fare riferimento a Fernanda Pivano, Lalla Romano, Lavinia Mazzucchetti, Alessandra e Liliana Scalero, Ada Prospero, Irene Riboni.

12. L. Di Nicola, Dalla parte dell'ombra. Donne e editoria, cit., p. 163.

13. Emblematica in tal senso è la definizione che Ginzburg dava di sé a Libero de Libero, che l'aveva descritta come una lettrice «severissima e intrattabile» in una lettera del 16 dicembre 1948: «io, invece, sono purtroppo una specie di pecora» (AE, fasc. De Libero). O ancora, in una lettera del 15 agosto 1946 a Pavese, Ginzburg scriveva: «hai l'aria di credere che noialtri si voglia diventare dei dirigenti; mentre nessuno più di me [...] aspira alla funzione di scopacessi» (AE, fasc. Ginzburg).

14. In Pro-memoria della direzione. Milano 6 agosto 1945, AE, fasc. Verbali editoriali, ora pubblicata in T. Munari (a cura di), I verbali del mercoledì. Riunioni editoriali Einaudi 1943-1952, Torino, Einaudi, 2011, pp. 46-48.

15. N. Saita, Natalia Ginzburg la fedeltà di una vita con «passo da soldato», cit., p. 97.

16. N. Ginzburg, È difficile parlare di sé, cit., p. 52

17. Amico dapprima di Leone, con cui aveva condotto gli studi al liceo D’Azeglio sotto la guida di Augusto Monti, Cesare Pavese frequenta la casa dei coniugi Ginzburg (come testimoniano diversi brani di Lessico famigliare) e stringe nel corso degli anni rapporti sempre più stretti anche con Natalia. Lo sgomento e la tristezza per la perdita dell'amico nell'agosto 1950 emergono in vari passi della produzione saggistica e narrativa di Ginzburg, in particolare in Lessico famigliare (ma si può già intravedere un tentativo di rielaborazione narrativa del suicidio di Pavese nel personaggio di Ippolito del romanzo Tutti i nostri ieri, uscito nel 1952) e in Ritratto di un amico (pubblicato dapprima, nel 1958, in «Radiocorriere-TV» e successivamente, nel 1962, nelle Piccole virtù). Per quanto riguarda il rapporto allieva-maestro, è la stessa Ginzburg a ricordare a più riprese quanto, da una parte, la propria narrativa fosse, soprattutto agli inizi, «influenzatissima da Pavese» (N. Ginzburg, È difficile parlare di sé, cit., p. 37; dello stesso avviso la critica: Cesare Garboli, amico e fine lettore dell'opera ginzburghiana, definirà questo romanzo di un «manierismo pavesiano-contadino» nell'introduzione all'edizione del 1993 ai Cinque romanzi brevi 
e altri racconti, Torino, Einaudi, prima ed.1964, p. viII) e dall'altra l'importanza e l'inestimabile valore del lavoro di Pavese presso Einaudi: «Dopo il suicidio di Pavese eravamo completamente persi, secondo me. Perché lui nella casa editrice faceva tutto; aveva una straordinaria facoltà lavorativa, io non ho mai visto una persona lavorare così tanto, fare tante cose. Secondo me aveva in mano la casa editrice; e oltre al grande dolore di averlo perduto, c'era questo senso di come avremmo fatto» (N. Ginzburg, È difficile parlare di sé, cit., p. 99). In quanto grande promotore delle opere ginzburghiane, Pavese propone ad esempio i testi della Ginzburg presso Giambattista Vicari, direttore di «Lettere d'oggi» (Cesare Pavese, Lettere 1924-1944, a cura di L. Mondo, Torino, Einaudi, 1966, pp.607-610) o presso Carlo Muscetta, collaboratore di «La Ruota» (C. Pavese, Officina Einaudi: lettere editoriali, 1940-1950, a cura di Silvia Savioli, p.9). Pavese è inoltre il responsabile, insieme a Mario Alicata, dell'esordio narrativo in casa editrice di Ginzburg, con La strada che va in città. È ancora una volta la stessa Ginzburg a sottolineare in tal senso il ruolo fondamentale di Cesare Pavese, raccontando di come ci sia proprio lui all'origine della pubblicazione del suo primo romanzo: «abbiamo avuto un permesso di dieci giorni e siamo a Torino; e io l'ho portato, manoscritto, l'ho dato a Pavese, e Pavese ha detto che lo pubblicava, sì, che gli andava bene, che lo pubblicava» (N. Ginzburg, È difficile parlare di sé, cit., p. 33).

18. C. Pavese, Officina Einaudi, cit., pp. 352-353. In merito alla condivisa concezione del lavoro editoriale, a titolo di esempio, si veda quando scritto in una nota per la Direzione da Ginzburg e Pavese, datata 2 ottobre 1947: «I due redattori-consulenti Natalia Ginzburg e Cesare Pavese, avendo constatato che il nuovo costume della Casa editrice tende a scansare ogni fatica redazionale e scaricarla sui fessi [...] dichiarano di appartenere anch'essi a quest'utile aristocrazia $\mathrm{e},[. .$.$] non toccando più né una bozza né un manoscritto, si daranno a fare romanzi e poesie, a$ legger classici e girare l'Italia» (AE, fasc. Pavese; ora pubblicata in L. Mangoni, Pensare i libri, cit., p. 449 n.).

19. AE, fascicolo Muscetta, ora in C. Pavese, Lettere 1945-1950, a cura di I. Calvino, Torino, Einaudi, 1966, p. 280.

20. C. Pavese, Officina Einaudi, cit., p. 352.

21. AE, fasc. Pavese, ora in C. Pavese, Lettere 1945-1950, cit., p. 356.

22. L. Mangoni, Pensare i libri, cit., p. 446.

23. Verbale della seduta editoriale del 12-13 gennaio 1949, AE, fasc. Verbali editoriali, ora pubblicata in T. Munari (a cura di), I verbali del mercoledì, cit., pp. 54-72.

24. AE, fasc. Pavese, ora in C. Pavese, Lettere 1945-1950, cit., p. 47.

25. AE, fasc. Pavese, ora in ivi, p. 105.

26. N. Ginzburg, È difficile parlare di sé, cit., pp. 88-89.

27. S. Cesari, Colloquio con Giulio Einaudi, cit., p. 162.

28. AE, fasc. Viganò, parzialmente pubblicato in L. Mangoni, Pensare i libri, cit., p. 448.

29. Ibid.

30. Si pensi alla recensione di Arrigo Cajumi, dedicata al Compagno di Pavese e a È stato così di Ginzburg («Il compagno», La Stampa, 21 agosto 1947) o a quella di Giorgio Bassani («Neorealisti italiani», in Lo spettatore italiano, I, 4, aprile 1948, pp.52-54, ora in G. Bassani, Opere, Milano, Mondadori, 1998, p. 1059). A distanza di anni, Cesare Garboli definirà "pavesiana e neorealista» (Introduzione a N. Ginzburg, Opere, vol. I, Milano, Mondadori, 1986, p. XXIII) tale fase creativa, in sostanziale accordo con la stessa autrice che, parlando del romanzo, sottolinea tuttavia la sua volontà di allontanarsi dai modi della narrativa pavesiana: «io lo scrivevo in una stanza, c'era Pavese che scriveva Il compagno [...]. Però io volevo un po' staccarmi dai modi di Pavese, volevo un po' sfuggire al suo influsso» (N. Ginzburg, È difficile parlare di sé, cit., p. 71).

31. N. Ginzburg, È difficile parlare di sé, cit., pp. 69-70.

32. N. Ginzburg, Lessico famigliare, Torino, Einaudi, 1963, pp. 165-167.

33. N. Ginzburg, È difficile parlare di sé, cit., p. 191. Sullo stesso tema, è utile citare almeno un passaggio di Senza una mente politica: «Io non credo che i romanzieri, e i romanzi che scrivono, 
custodiscano dentro di sé dei messaggi politici, destinati a rendere migliore il mondo. Non credo che i romanzieri, e i romanzi che scrivono, possano mai essere utili alla vita pubblica. Credo fermamente nella loro splendida, meravigliosa, libera inutilità» (ora in N. Ginzburg, Non possiamo saperlo, Saggi 1973-1990, Torino, Einaudi, 2001, p. 105).

34. Cfr. N. Ginzburg, È difficile parlare di sé, cit., p. 71.

35. AE, fasc. Invrea. La prima stesura di cui parla Ginzburg, come testimoniato dai documenti d'archivio, risale al 1947.

36. AE, fasc. Vittorini.

37. AE, fasc. Pavese, ora in C. Pavese, Lettere 1945-1950, cit., p. 280.

38. AE, fasc. Ginzburg, parzialmente pubblicato in L. Mangoni, Pensare i libri, cit., p. 470.

39. AE, fasc. Vittorini.

40. AE, fasc. Ginzburg, parzialmente pubblicato in G. Iannuzzi, Natalia Ginzburg, cit., p. 126.

41. Ibid. Il libro uscirà poi per Le edizioni europee nel 1949.

42. AE, fasc. Antonielli, ora in V. Camerano, R. Crovi e G. Grasso (a cura di), La storia dei «Gettoni» di Elio Vittorini, vol. II, Torino, N. Aragno, 2007, pp. 839-840.

43. AE, fasc. Vittorini, parzialmente pubblicato in L. Mangoni, Pensare i libri, cit., p. $452 \mathrm{n}$.

44. AE, fasc. Ginzburg, ora in M. A. Grignani e D. Scarpa (a cura di), Natalia Ginzburg, cit., p. 191.

45. AE, fasc. Ginzburg, ora in ivi, p. 192.

46. N. Ginzburg, È difficile parlare di sé, cit., p. 112.

47. N. Ginzburg, Il mio mestiere, in «Il Ponte», vol. V, no 8-9, agosto-settembre 1949, p. 1194, pubblicato successivamente in Le piccole virtù, cit., p. 55-69.

48. N. Ginzburg, Cinque romanzi brevi e altri racconti, Torino, Einaudi, 1993 (1964), p. 17.

49. Ivi, p. 8.

50. Ivi, p. 18.

\section{RIASSUNTI}

L'obiettivo del saggio è quello di mettere in luce l'importanza del ruolo di Natalia Ginzburg presso Einaudi nel dopoguerra, analizzando la sua funzione all'interno dei processi decisionali, nella costruzione dell'identità e del catalogo della casa editrice e, più in generale, nella definizione della cultura letteraria del suo tempo, con un'attenzione particolare dedicata all'attività di lettura editoriale. Lo studio dei pareri di lettura redatti da Natalia Ginzburg per le collane di narrativa costituisce la base della riflessione sulla sua attività editoriale, in particolar modo come lettrice, nella convinzione che la scrittura editoriale rappresenti un genere critico particolare e complesso.

L'objectif de l'article est d'étudier l'activité de lecture éditoriale de Natalia Ginzburg chez Einaudi dans l'après-guerre, en analysant de quelle manière elle a contribué aux processus décisionnels, à la construction de l'identité et du catalogue de la maison d'édition et, plus généralement, à la définition de la culture littéraire de son époque, une attention particulière étant dédiée à la lecture éditoriale. L'étude des rapports de lecture qu'elle rédigea pour les collections d'œuvres narratives fournit les bases d'une réflexion sur son activité éditoriale, notamment en tant que lectrice professionnelle, à partir de notre conviction que l'écriture éditoriale représente un genre critique particulier et complexe méritant d'être étudié par des méthodes spécifiques. 
The main objective of this essay is to highlight the importance of Natalia Ginzburg's role at Einaudi's in the aftermath of World War II. The emphasis is placed on her contribution to decision-making processes, to the construction of the identity and of the catalogue of the publishing house and, more generally, to the definition of the literary culture of her time, with particular attention to editorial reading. The examination of her reading reports, mostly unpublished, provides the basis for a critical reflection on Ginzburg's editorial activity, a reflection which is rooted in the belief that editorial writing represents a particular and complex critical genre that deserves to be investigated by specific methods.

\section{INDICE}

Parole chiave : Natalia Ginzburg, case editrici, Einaudi, lettura editoriale, pareri di lettura Mots-clés : Natalia Ginzburg, maisons d'édition, Einaudi, lecture éditoriale, rapports de lecture Keywords : Natalia Ginzburg, publishing houses, Einaudi, editorial reading, reading reports

\section{AUTORE}

\section{LAURA ANTONIETTI}

Université Grenoble Alpes

laura.antonietti@univ-grenoble-alpes.fr 